\section{Der Osteologe DVO: eine echte Erfolgsgeschichte}

D ie rasche Zunahme der Kenntnisse in Diagnostik und Therapie von Knochenstoffwechselerkrankungen, insbesondere der Osteoporose, führte bereits 1984 zur Gründung der Deutschen Gesellschaft für Osteologie. Da diese zunächst internistisch dominiert war, erfolgte als Plattform aller orthopädischen Osteologen die Gründung der Orthopädischen Gesellschaft für Osteologie (OGO).

Die zunehmende Bedeutung der „Osteologie“ erkannte auch die Deutsche Gesellschaft für Orthopädie und Traumatologie (DGOT), weshalb am 01.05.1999 im Rahmen der Jahrestagung der Vereinigung Süddeutscher Orthopäden die Sektion "Osteologie“ der Allianz Deutscher Orthopäden gegründet wurde. Ziele der Sektion waren damals und wie heute eine Verbesserung der wissenschaftlichen Arbeit auf dem Gebiet der Osteologie innerhalb der Orthopädie, eine Förderung und Koordinierung der osteologischen Grundlagenforschung sowie eine Stärkung des Interesses an Diagnostik und Therapie osteologischer Erkrankungen innerhalb der Orthopädie. Die Sektion Osteologie und die OGO arbeiten hierzu eng zusammen. Um die osteologische Fortbildung zu aktivieren, werden seit 2000 auf dem Deutschen Orthopädenkongress als auch auf den Jah- restagungen der Vereinigung Süddeutscher Orthopäden Osteologieseminare $\mathrm{zu}$ verschiedensten osteologischen Themen angeboten. Ab dem Jahr 2002 waren die osteologischen Seminare auch Bestandteil der Osteologiekongresse.

Die Seminarthemen wurden in der Buchreihe „Fortbildung Osteologie“ publiziert. Drei Bände sind bereits erschienen, ein Vierter befindet sich in der finalen Vorbereitungsphase. Der Osteologiekongress in Graz 2002 war auch die Geburtsstunde der Zertifizierungskurse zum Osteologen DVO.

Als Mitglieder des inzwischen gegründeten Dachverbandes Osteologie hatten die OGO als auch die Sektion Osteologie aktiv sowohl an der Verabschiedung der ersten Osteoporoseleitlinien als auch an der Gestaltung der Osteologiekurse mitgewirkt.

Der erste Osteologiekurs fand 2002 in der Dr. Becker Rhein-Sieg-Klinik in Nümbrecht statt. Da die Zahl der an der Osteologie interessierten Fachärzte bundesweit auf max. 200 bis 250 eingeschätzt wurde, bestand ein erhebliches Risiko, für einen Osteologiekurs überhaupt genügend Teilnehmer zusammen fiel deshalb auf Nümbrecht, da ich als Vorsitzender der Sektion Osteologie eine kostenfreie Nutzung der Räumlichkeizu bekommen. Die Wahl des Kursortes

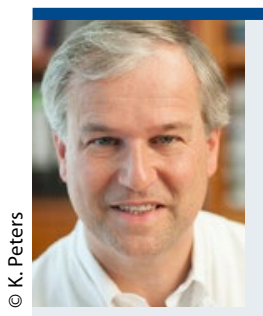

Prof. Dr. med. Klaus

M. Peters

Orthopädie und Osteologie,

Dr. Becker Rhein-Sieg-Klinik, Nümbrecht

ten in der Dr. Becker Rhein-Sieg-Klinik zusagen konnte. Es wurde gehofft, 40 bis 50 Teilnehmer generieren zu können. In der Realität war der erste Osteologiekurs dann bereits nach wenigen Tagen mit über 100 Teilnehmern mehr als ausgebucht. Teilnehmer aus ganz Deutschland strömten nach Nümbrecht. Im Jahr 2003 fand dann eine erste Kursreihe mit Zertifizierungskursen I-III in Nümbrecht und Würzburg statt.

Das Interesse an der Osteologie ist auch 14 Jahre später ungebrochen. Es finden weiterhin regelmäßig Zertifizierungsals auch Rezertifizierungskurse unter der organisatorischen Leitung der Osteologie-Akademie (OSTAK) statt.

Auch wenn die Osteologie bis heute keine Zusatzbezeichnung der Ärztekammern ist, steht die Bezeichnung „Osteologe DVO“ hoch im Kurs und ist Voraussetzung zur Teilnahme an IV-Verträgen Osteoporose.

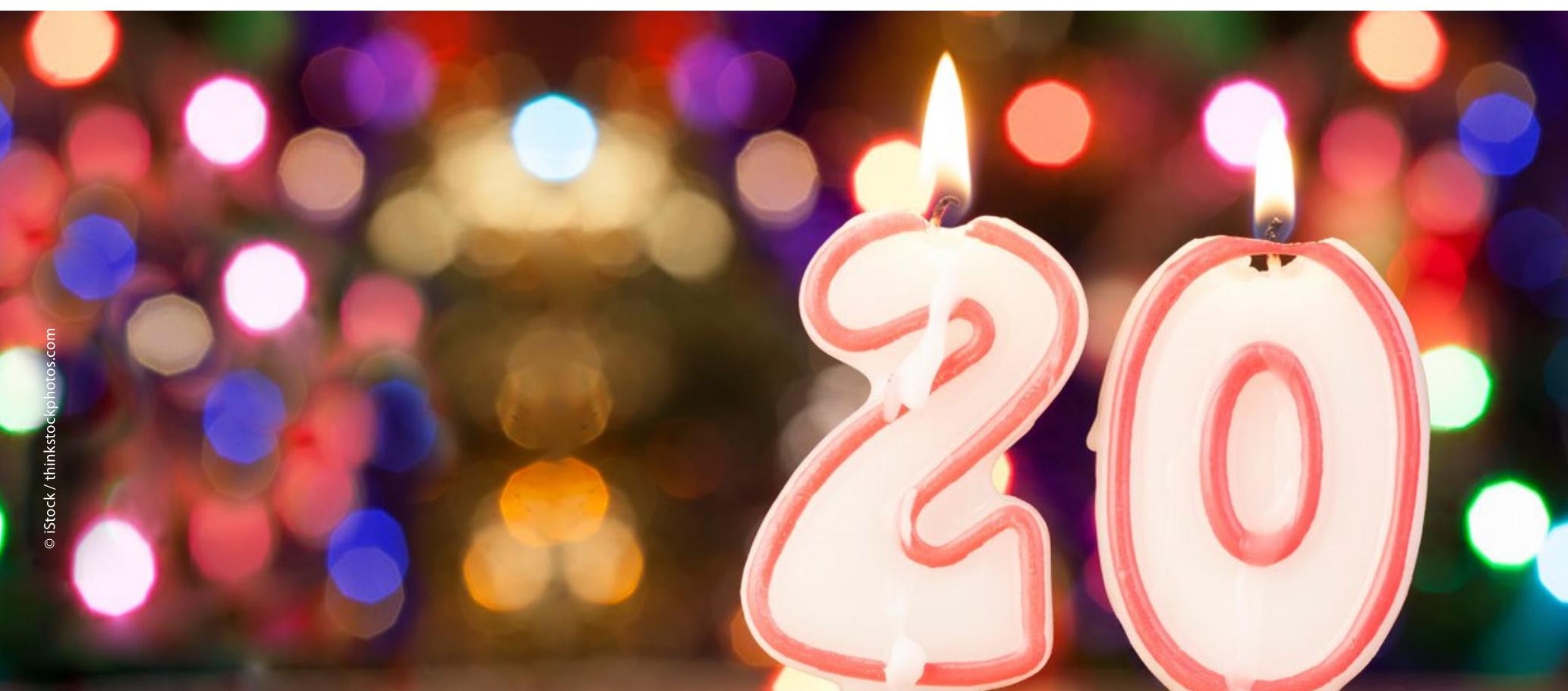

